\title{
Wideband linear detector arrays for optoacoustic imaging based on polyvinylidene difluoride films
}

\author{
Pavel Subochev \\ Maxim Prudnikov \\ Vladimir Vorobyev \\ Anna Postnikova \\ Egor Sergeev \\ Valeria Perekatova \\ Anna Orlova \\ Valentina Kotomina \\ Ilya Turchin
}




\title{
Wideband linear detector arrays for optoacoustic imaging based on polyvinylidene difluoride films
}

\author{
Pavel Subochev, ${ }^{\mathrm{a}, \star}$ Maxim Prudnikov, ${ }^{\mathrm{a}}$ Vladimir Vorobyev, ${ }^{\mathrm{a}}$ Anna Postnikova, ${ }^{\mathrm{a}}$ Egor Sergeev, ${ }^{\mathrm{a}}$ \\ Valeria Perekatova, ${ }^{a}$ Anna Orlova, ${ }^{a}$ Valentina Kotomina, ${ }^{b}$ and llya Turchin ${ }^{a}$ \\ anstitute of Applied Physics RAS, Nizhny Novgorod, Russia \\ bobachevsky State University of Nizhny Novgorod, Nizhny Novgorod, Russia
}

\begin{abstract}
We provide direct experimental comparison of the optoacoustic imaging performance of two different 64-element linear detector array (LDA) units based on polyvinylidene difluoride (PVDF) films. The first LDA unit was based on traditional flexible circuit (FC) technology and consisted of an FC glued to the nonmetalized signal surface of a $28-\mu \mathrm{m}$-thick PVDF film providing 300/80- $\mu \mathrm{m}$ axial resolution/lateral resolution (AR/LR) and 0.4-kPa noise equivalent pressure of its single element. The other LDA unit was manufactured using a technology of low-temperature photolithographic etching (PE) of a signal electrode onto a $25-\mu \mathrm{m}$-thick PVDF film providing $300 / 40-\mu \mathrm{m} \mathrm{AR/LR}$ and $1 \mathrm{kPa}$ noise equivalent pressure. As compared with a previously reported LDA unit based on a 100- $\mu \mathrm{m}$ PVDF thick film, the main advantage of using the thinner PVDF films was 10-fold improvement in axial resolution, whereas the main drawback was 10-fold increased noise equivalent pressure. In terms of in vivo imaging performance, higher bandwidth of PE LDA probe was more important than the higher sensitivity of FC LDA unit. @ 2018 Society of Photo-Optical Instrumentation Engineers (SPIE) [DOI: 10.1117/1.JBO.23.9.091408]
\end{abstract}

Keywords: optoacoustic tomography; polyvinylidene difluoride films; linear detector arrays.

Paper 170784SSRR received Dec. 5, 2017; accepted for publication May 1, 2018; published online May 22, 2018.

\section{Introduction}

Biomedical optoacoustic (OA) imaging is based on the remote detection of ultrasonic signals generated in biological tissue as a result of the absorption of pulsed laser radiation by optical heterogeneities. ${ }^{1}$ Recent advances in the manufacturing technology for pulsed tunable lasers have enabled the development of video-rate multispectral OA tomography systems. ${ }^{2}$ A commonly quoted clinical application strategy for the use of OA methods ${ }^{3}$ is based on the ability to integrate OA imaging equipment into clinical ultrasound (US) scanners. ${ }^{4}$ However, the limited bandwidth of conventional piezoelectric US linear detector array (LDA) transducers does not allow effective use of these for the detection of broadband OA pulses. ${ }^{5}$

To widen the detection bandwidth, alternative LDA units based on polyvinylidene difluoride (PVDF) have been introduced $^{6}$ to biomedical OA imaging. ${ }^{7}$ The successful integration of a wideband LDA unit based on 100- $\mu$ m PVDF film with a commercial US scanner was reported. ${ }^{8}$ The drawback of PVDF film is low efficacy of piezoelectric conversion that challenges electrical generation of probing US pulse. Nevertheless, to realize US imaging capabilities of PVDF-based arrays, it is possible to use optical generation of probing US pulses. ${ }^{9}$

Our study is devoted to further improvement in the detection bandwidth provided by two PVDF LDA units. Both units are tested in phantom and in vivo experiments. The first LDA unit is also based on the flexible circuit (FC) technology; ${ }^{8}$ however, a thinner $28-\mu \mathrm{m}$ PVDF film is used. The second LDA unit is based on the low-temperature photolithographic

*Address all correspondence to: Pavel Subochev, E-mail: Pavel.Subochev@ gmail.com etching (PE) of a signal electrode on a $25-\mu \mathrm{m}$ PVDF film. The newly introduced PE technology allows using a backing material matching the acoustic impedance of PVDF, thus providing a further improvement in the LDA bandwidth.

\section{Materials and Methods}

\subsection{Flexible Circuit Linear Detector Array}

The fabrication technology of the FC LDA unit was similar to the one described. ${ }^{8}$ However, a much thinner $28-\mu \mathrm{m}$ PVDF film (Precision Acoustics, UK) was used to improve the bandwidth.

The nonmetalized signal surface of the PVDF film was glued to an FC consisting of 64 electrodes corresponding to a $300-\mu \mathrm{m}$ lateral pitch and a 75- $\mu \mathrm{m}$ kerf width [Fig. 1(b)]. Then, the flexon-PVDF stack was fixed against a cylindrical block defining 25-mm elevation focus. The inner housing was filled with a compound having an acoustic impedance of 4 MRayl, corresponding to the acoustic impedance of the PVDF film.

\subsection{Photolithographically Etched Linear Detector Array}

The PE LDA unit was manufactured using a $25-\mu \mathrm{m}$-thick PVDF film (Precision Acoustics, UK). The PE procedure was applied to the gold signal electrode of the PVDF film. To avoid irreversible degradation of the performance of the PVDF film, the entire photolithographic process was performed at temperatures below $80^{\circ} \mathrm{C}$. The etching of the gold layer was carried out in an etchant of composition $\mathrm{J} / \mathrm{KJ} / \mathrm{H}_{2} \mathrm{O}$ with a component ratio of $1 \mathrm{~g} / 5 \mathrm{~g} / 20 \mathrm{ml}$.

$1083-3668 / 2018 / \$ 25.00$ @ 2018 SPIE 

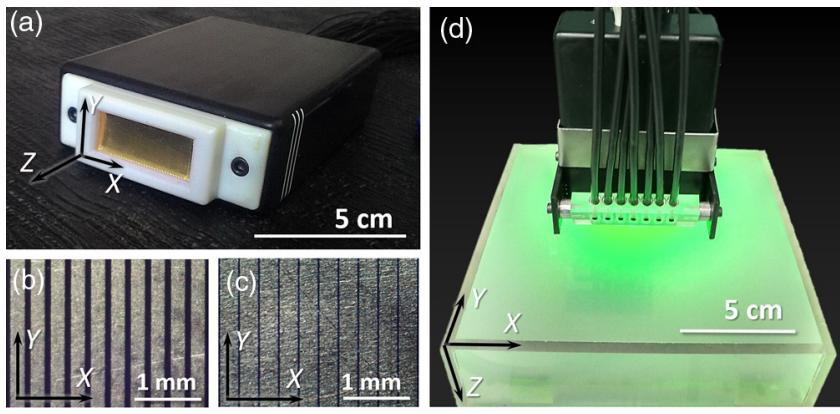

Fig. 1 LDAs for optoacoustic tomography based on PVDF films: (a) photographic image of FC LDA, (b, c) lateral geometry of signal electrodes for FC LDA and PE LDA, and (d) the geometry of experiment with black half-space phantom.

After 64 strips with a $300-\mu \mathrm{m}$ lateral pitch and $25-\mu \mathrm{m}$ kerf width [Fig. 1(c)] had been etched, the PVDF film was clamped to the cylindrical block defining 25 -mm elevation focus, with the etched side uppermost. The signal contacts at both sides of the inner housing were connected with two 32-channel auxiliary FCs using conducting silver-loaded epoxy. The spatial period of the auxiliary FCs was $600 \mu \mathrm{m}$. The inner housing was placed on top and filled with a compound with an acoustic impedance of $4 \mathrm{MRayl}$, corresponding to the acoustic impedance of the PVDF film.

\subsection{Impedance-Matching Amplifier}

Each of the 64 channels consisted of an MMBFJ310 (Fairchild Semiconductors) double-gate field effect transistor, used as an amplifier, and a BFR505 (Texas Instruments) bipolar transistor functioning as a repeater for matching the amplifier with the low-resistance load $(50 \mathrm{Ohm})$. The impedance-matching performance was secured by the small throughput capacity of the double-gate transistor; the resulting cutoff frequency at high frequencies was more than $50 \mathrm{MHz}$. The amplifier transmission coefficient was in the range of 3.5 to 4 (because of the spread of the transistor parameters). Due to the small $2 \mathrm{pF}$ input capacitance of the MOS transistor, the operating current of each amplifying channel was about $12 \mathrm{~mA}$.

After interconnection of the impedance-matching 64-channel amplifier with the signal and ground electrodes of the corresponding LDA unit, it was placed into the shielding housing and the outer casing, containing the elements for sealing and fastening [Fig. 1(a)].

\subsection{Experimental Setup}

A photograph of the experimental setup during the phantom experiment is shown in Fig. 1(d).

Fourteen output arms of a fiber bundle (Ceram Optec, Germany) were placed at each side of the LDA unit and were directed onto the elevation focus [Fig. 1(d)]. Each arm was $2.5 \mathrm{~mm}$ in diameter with a 0.17 numerical aperture in water. Laser pulses at a wavelength of $532 \mathrm{~nm}$ with a pulse duration of 16 ns were provided by an LT-2214-PC (LOTIS TII, Belorussia) solid-state laser with a $10-\mathrm{Hz}$ pulse repetition rate. A custom-made 10:90 beam splitter with a calibrated ES111C (Thorlabs) pyroelectric sensor was used to determine the energy of each single laser pulse at the distal fiber tips.

For axial resolution (AR) study, a single-channel ADC based on an NI5761 14-bit adapter with an NI FlexRIO FPGA PXI-
7952R module (National Instruments) was connected to the central element of each LDA. The sample rate of A-scans was $250 \mathrm{MS} / \mathrm{s}$. For lateral resolution (LR) study, the central element of each LDA unit was scanned against the phantom using a linear positioning slide (Automation Gages) and an AM-23-239-3 step motor (Advanced Microsystems). The lateral scanning steps $64 \times 300 \mu \mathrm{m}$ matched the lateral pitch of both LDA units.

For in vivo study, 16 ADC channels based on four NI5761 14-bit adapters (National Instruments) were multiplexed with 64 elements of each LDA using 16 ADG711 switchers (Analog Devices). The sample rate of A-scans was $125 \mathrm{MS} / \mathrm{s}$.

\subsection{Axial and Lateral Resolution}

Both FC LDA and PE LDA units were subsequently fixed on top of a cuvette filled with a $1 \%$ aqueous solution of lypofundin. A strip of black polyester film (Orafol) of width $50 \mathrm{~mm}$ and thickness $80 \mu \mathrm{m}$ was placed at the elevation focus.

To estimate the AR of the FC LDA and PE LDA units, the impulse response of each LDA unit to the back-scattered laser pulse ${ }^{9}$ was used. To balance the lower and higher frequency contents of the LDA units, a 5-MHz high-pass filter was applied to raw A-scan. Every raw A-scan was subjected to Hilbert's transform, normalized to the energy of its corresponding laser pulse, and normalized to the standard deviation of the noise.

To estimate the LR of the FC LDA and PE LDA units, the reconstructed images of the absorbing strip were measured by each LDA unit. Reconstructed B-scans $I_{\text {Recon }}$ were obtained by applying a Fourier reconstruction algorithm ${ }^{10}$ to the raw OA B-scans (consisting of 64 A-scans). To improve the smoothness of the reconstructed $\mathrm{B}$-scans in the $x$-direction, interpolations of the A-scans were added before reconstruction. ${ }^{10}$ The reconstructed $\mathrm{OA}$ intensities at all $(X, Z)$ points within the $I_{\text {Recon }}$ and $I_{\text {Interp }}$ images for both LDA units were normalized to the corresponding standard deviations of the noise. To estimate the relative signal-to-noise ratio (SNR) of the FC LDA and PE LDA units, the maximum intensity projections (MIPs) of the $I_{\text {Interp }}$ images to the $x$-axis were calculated (the edge spread functions). The spatial derivative of MIP provided the line spread function (LSR). ${ }^{11}$ The LR of FC LDA and PE LDA units was, therefore, estimated as full width at half maximum of LSR.

\subsection{In Vivo Imaging and the Noise Equivalent Pressure}

The animal subject (Continental Giant White rabbit, 6-kg body weight) used for in vivo imaging was handled in accordance with international rules of legal and ethical use of animals. Before the investigation, the rabbit was anaesthetized with an intramuscular injection of a mixture of $40 \mathrm{mg} / \mathrm{kg}$ of Zoletil 100 with $1.5 \mathrm{mg} / \mathrm{kg}$ Rometar. The selected region of the rabbit's ear was oriented parallel to the $X Z$ plane, both LDAs were focused to the same blood vessel parallel to the $\mathrm{X}$-axis. The reconstructed B-scans $I_{\text {Recon }}$ of the rabbit's ear were obtained by applying a Fourier reconstruction algorithm ${ }^{10}$ to the raw OA B-scans (consisting of 64 A-scans).

Raw B-scans of the rabbit's ear were acquired at $\Phi=$ $20 \mathrm{~mJ} / \mathrm{cm}^{2}$ irradiance at 532-nm wavelength. Assuming = 0.16 Gruneisen coefficient and $\mu_{a}=22 \mathrm{~mm}^{-1}$ blood optical absorption, the initial pressure was estimated as $P_{0}=704 \mathrm{kPa}$. Three-dimensional K-wave model ${ }^{12}$ matching the geometry of the in vivo experiment allowed to calculate the maximum 
pressure at the central element of each LDA unit as $P_{\max }=$ $39 \mathrm{kPa}$. To estimate the noise equivalent pressure (NEP), maximum amplitudes of raw in vivo B-scans were scaled to $P_{\max }=39 \mathrm{kPa}$ value.

\section{Results and Discussion}

Figures 2(a) and 2(b) show the impulse responses of the FC LDA and PE LDA units to the scattered laser pulse ${ }^{9}$ normalized to the standard deviation of the noise for each LDA unit. According to the theory, ${ }^{13}$ one-dimensional (1-D) interaction of the laser pulse with PVDF film should provide the impulse response with unipolar profile.

The shortest impulse response with almost unipolar profile is provided by PE LDA unit [Fig. 2(b)]. The FC LDA unit demonstrates resonant behavior in time domain [Fig. 2(a)] and rather inhomogeneous power spectral density (PSD) [Fig. 2(c)]. The PE LDA unit provides a more accurate balance of the lower and higher frequencies [Fig. 2(d)] and has a higher cutoff frequency, of at least $40 \mathrm{MHz}$.

Figures 3(a) and 3(b) show the raw OA B-scans (each consisting of 64 A-scans) acquired from the thin black plastic strip with its edge located at the elevation focus of the FC LDA and
PE LDA units. Figures 3(c)-3(f) show the reconstructed OA intensities when the reconstruction procedure is applied to either the raw OA B-scans [Figs. 3(a) and 3(b)] or to the interpolated B-scans (not shown). Figures 3(g) and 3(h) show the MIPs of the OA images [Figs. 3(e) and 3(f)] to the x-axis. The FC LDA unit [Fig. 3(g)] provides a twofold improvement in SNR compared with the PE LDA ones [Fig. 3(h)].

Figure 4 characterizes the spatial resolution of the FC LDA and PE LDA units. The AR for both LDA units [Figs. 4(a) and 4(b)] was estimated as full width at half maximum (FWHM) for the envelope of each corresponding impulse response [Figs. 2(a) and 2(b)]. The LR for both LDA units [Figs. 4(c) and 4(d)] was estimated as FWHM for the derivative of each corresponding MIP [Figs. 3(g) and 3(h)].

The twofold differences in the ARs are related to the metal electrodes of the FC being located at the signal electrode of the PVDF film in the case of the FC LDA unit. The metal backing of the FC LDA efficiently reflects the acoustic signals backward through the same thickness of the PVDF film, thus providing a prolonged piezoelectric transformation of each given acoustic pulse at the cost of an increased impulse response. The PE LDA unit was not affected by the same phenomenon as the acoustic
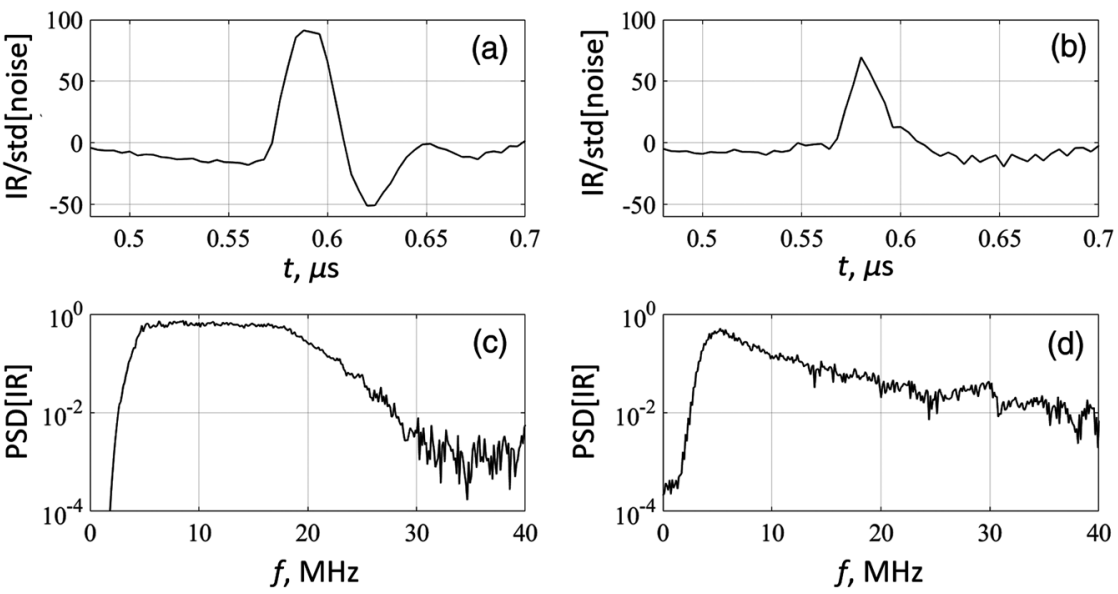

Fig. 2 Impulse response of central element for FC LDA (left column) and PE LDA (right column) after $5-\mathrm{MHz}$ high-pass filtration: $(a, b)$ time series normalized to standard deviation of noise, (c, d) PSD.
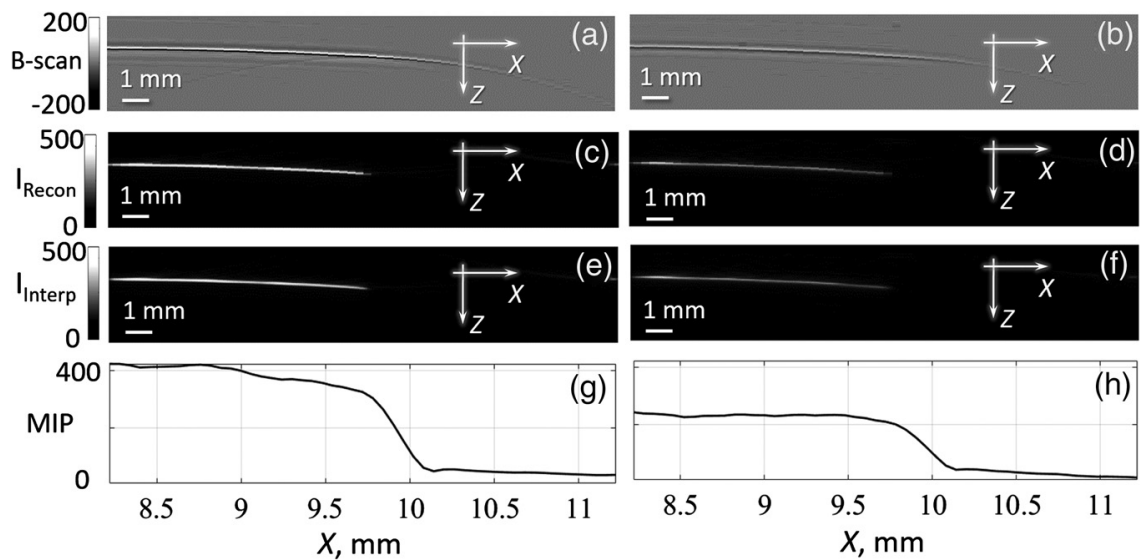

Fig. 3 Optoacoustic imaging of a half-space phantom by FC LDA (left column) and PE LDA (right col$\mathrm{umn})$ : (a, b) raw B-scans normalized to standard deviations of noise, $(c, d)$ reconstructed intensities normalized to standard deviations of noise, $(e, f)$ interpolated and reconstructed intensities normalized to standard deviations of noise, and $(\mathrm{g}, \mathrm{h})$ projections of maximum intensities to the $x$-axis (lateral profile of half-space phantom). 

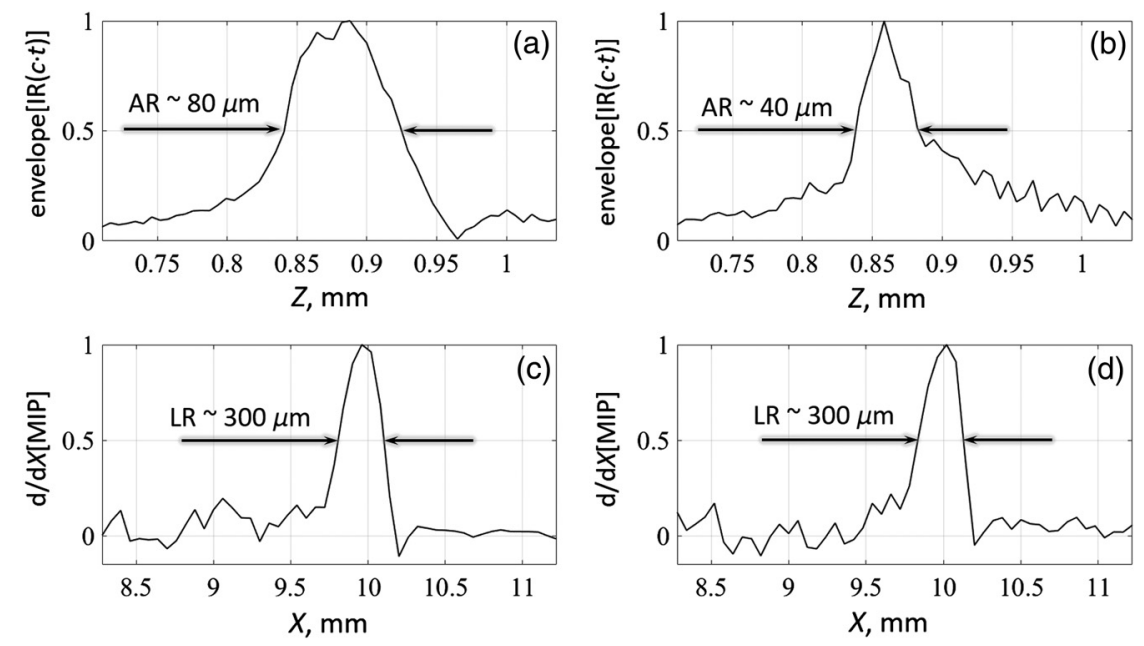

Fig. 4 Spatial resolution of FC LDA (left column) and PE LDA (right column): (a, b) AR, as the effective spatial scale of impulse response, (c, d) LR, as the derivative from lateral profile of half-space phantom.

impedance of PE LDA backing directly matched the acoustic impedance of the PVDF film. The identical LRs of the FC LDA and the PE LDA can be explained by the equal $300-\mu \mathrm{m}$ pitch of each.

Figures 5(a) and 5(b) show the superior imaging performance of PE LDA during the in vivo experiment. The larger amount of reconstruction artifacts at Fig. 5(a) is consistent with the narrower bandwidth of FC LDA unit [Fig. 2(c)]. Both LDAs allow visualizing the major blood vessels oriented in the $X$-direction; however, the vasculature oriented in the Z-direction is not visualized due to the limited numerical aperture (NA 0.3) of both arrays. Limited-view artifacts can be reduced either by scanning the investigated sample ${ }^{14}$ or by employing toroidal PVDF sensor arrays, ${ }^{15}$ instead of cylindrical ones.

The raw A-scans represented at Figs. 5(c) and 5(d) allowed to estimate the NEP of both arrays, as the standard deviation of noise. Unfortunately, due to the high input impedance and ultrawide bandwidth of an amplifier the major contribution to NEP was provided by electromagnetic interference, rather than the thermal noise. The higher NEP of PE LDE probe [Fig. 5(d)] is consistent with its lower SNR observed in phantoms experiment [Fig. 3(h)]. The resulting NEPs (Fig. 5) are comparable to NEP of the wideband photoacoustic detector array based on a 2-D Fabry-Perot interferometer $(0.2 \mathrm{kPa}) .{ }^{16}$ However, the technologies of single-element purely optical detection of an US are generally much more superior allowing NEPs of 2 to $20 \mathrm{~Pa}^{17,18}$

As compared with an earlier reported PPA unit ${ }^{8}$ based on a $100-\mu$ m-thick PVDF film, our PE LDA and FC LDA units are based on a 25 - and $28-\mu$ m-thick PVDF films. The main advantage of using the thinner PVDF film is an improved AR $(420 \mu \mathrm{m}$ for PPA, ${ }^{8} 40 \mu \mathrm{m}$ for PE LDA, and $80 \mu \mathrm{m}$ for FC LDA). The main drawback of using the thinner films is an increased noise equivalent pressure $\left(0.1 \mathrm{kPa}\right.$ for $\mathrm{PPA},{ }^{8} 0.4 \mathrm{kPa}$ for $\mathrm{FC}$ LDA, and $1 \mathrm{kPa}$ for PE LDA). In terms of in vivo OA imaging performance, the higher spectral sensitivity of PE LDA probe in
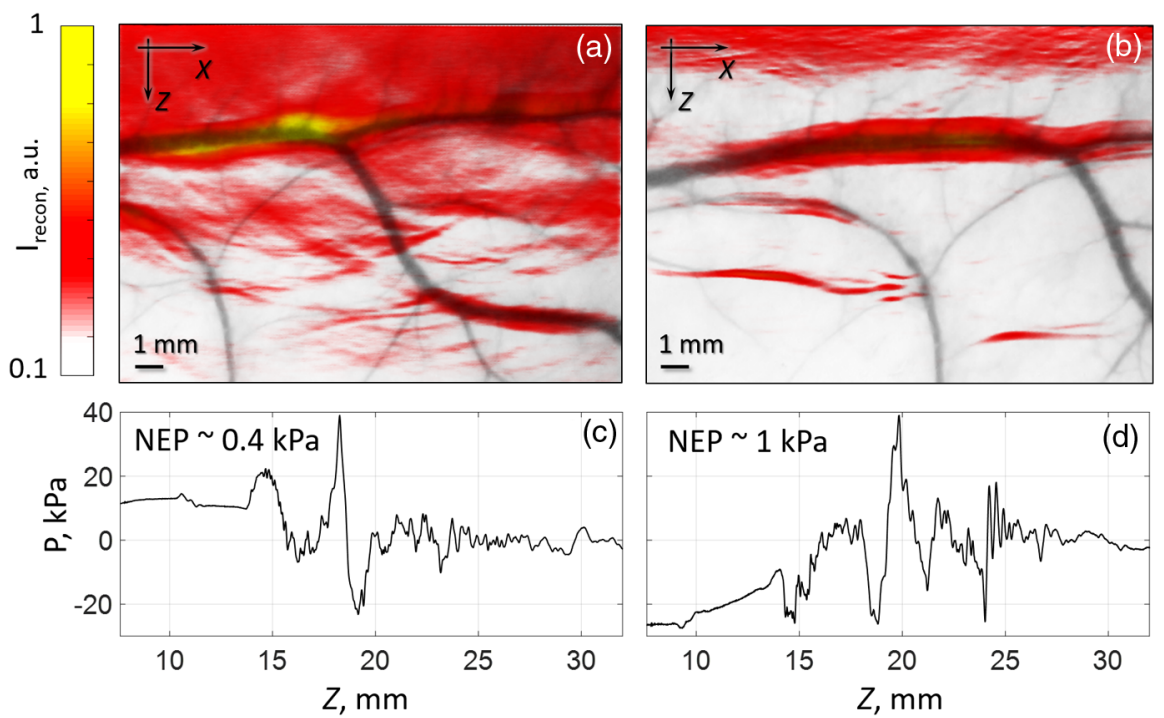

Fig. 5 In vivo OA imaging of rabbit's ear using FC LDA (left column) and PE LDA (right column): (a, b) reconstructed $\mathrm{OA}$ images of vasculature (color scale) on top of transillumination photography of the blood vessels (gray scale), (c, d) raw A-scans of the same blood vessel scaled to $P_{\max }=39 \mathrm{kPa}$. 
25- to 40-MHz range (Fig. 2) is more important than the higher overall SNR of FC LDA unit in the time domain (Fig. 3). The nonresonant nature of PE LDA impulse response significantly reduces the amount of interference artifacts at the reconstructed in vivo OA images (Fig. 5).

\section{Disclosures}

The authors can confirm that they have no conflicts of interest.

\section{Acknowledgments}

The work was supported by Russian Science Foundation (project 14-15-00709). The authors are grateful to Roman Belyaev, Sergey Pozhidaev, and Alexey Kharitonov for their engineering contributions to this work; to Dr. Michael Jaeger for provision of the reconstruction algorithm; to Dr. Andrew Hurrell and to Dr. Ivan Pelivanov for their valuable contribution to discussions.

\section{References}

1. L. V. Wang and J. Yao, "A practical guide to photoacoustic tomography in the life sciences," Nat. Methods 13(8), 627-638 (2016).

2. X. L. Deán-Ben et al., "Functional optoacoustic neuro-tomography for scalable whole-brain monitoring of calcium indicators," Light Sci. Appl. 5(12), e16201 (2016).

3. S. Zackrisson, S. van de Ven, and S. Gambhir, "Light in and sound out: emerging translational strategies for photoacoustic imaging," Cancer Res. 74(4), 979-1004 (2014).

4. R. Bouchard, O. Sahin, and S. Emelianov, "Ultrasound-guided photoacoustic imaging: current state and future development," IEEE Trans. Ultrason. Ferroelectr. Freq. Control 61(3), 450-466 (2014).

5. S. Preisser et al., "Study of clutter origin in in-vivo epi-optoacoustic imaging of human forearms," J. Opt. 18(9), 094003 (2016).

6. F. S. Foster, K. A. Harasiewicz, and M. D. Sherar, "A history of medical and biological imaging with polyvinylidene fluoride (PVDF) transducers," IEEE Trans. Ultrason. Ferroelectr. Freq. Control 47(6), 13631371 (2000).

7. T. D. Khokhlova et al., "Optoacoustic imaging of absorbing objects in a turbid medium: ultimate sensitivity and application to breast cancer diagnostics," Appl. Opt. 46(2), 262-272 (2007).

8. X. Wang et al., "Photoacoustic imaging with a commercial ultrasound system and a custom probe," Ultrasound Med. Biol. 37(3), 484-492 (2011).

9. P. Subochev et al., "Simultaneous in vivo imaging of diffuse optical reflectance, optoacoustic pressure, and ultrasonic scattering," Biomed. Opt. Express 7(10), 3951-3957 (2016).

10. M. Jaeger et al., "Fourier reconstruction in optoacoustic imaging using truncated regularized inverse k-space interpolation," Inverse Prob. 23(6), S51-S63 (2007).

11. J. Chen et al., "Blind-deconvolution optical-resolution photoacoustic microscopy in vivo," Opt. Express 21(6), 7316-7327 (2013).

12. B. E. Treeby and B. T. Cox, "k-Wave: MATLAB toolbox for the simulation and reconstruction of photoacoustic wave fields," J. Biomed. Opt. 15(2), 021314 (2010).

13. G. Diebold, T. Sun, and M. Khan, "Photoacoustic monopole radiation in one, two, and three dimensions," Phys. Rev. Lett. 67(24), 3384-3387 (1991).

14. Y. Wang et al., "Toward whole-body quantitative photoacoustic tomography of small-animals with multi-angle light-sheet illuminations," Biomed. Opt. Express 8(8), 3778-3795 (2017).

15. A. S. Bychkov et al., "Toroidal sensor arrays for real-time photoacoustic imaging," J. Biomed. Opt. 22(7), 076003 (2017).
16. E. Zhang, J. Laufer, and P. Beard, "Backward-mode multiwavelength photoacoustic scanner using a planar Fabry-Perot polymer film ultrasound sensor for high-resolution three-dimensional imaging of biological tissues," Appl. Opt. 47(4), 561-577 (2008).

17. Z. Xie et al., "Pure optical photoacoustic microscopy," Opt. Express 19(10), 9027-9034 (2011).

18. S. Preisser et al., "All-optical highly sensitive akinetic sensor for ultrasound detection and photoacoustic imaging," Biomed. Opt. Express 7(10), 4171-4186 (2016).

Pavel Subochev graduated from Lobachevsky State University of Nizhni Novgorod in 2006 and received his PhD in passive acoustic radiometry from the Institute of Applied Physics of Russian Academy of Sciences. He is an optoacoustic group leader of the Laboratory of Biophotonics of the Institute of Applied Physics RAS. Since 2012, he is focused on the development of new instrumentation for optoacoustic imaging of biological tissues. He is the coauthor of 15 peer-review articles related to optoacoustic.

Maxim Prudnikov is a leading mechanical engineer of Laboratory of Biophotonics of the Institute of Applied Physics RAS. More than 30 years of expertise in the development of custom-made ultrasonic detectors for biomedical applications. He is a coauthor of several patents.

Vladimir Vorobyev is an electrical engineer of Laboratory of Biophotonics of the Institute of Applied Physics RAS. More than 30 years of expertise in building custom-made radioelectronic devices for various biomedical applications. He is a coauthor of several patents.

Anna Postnikova is a senior scientist at Department of Automation of Scientific Research of the Institute of Applied Physics RAS. More than 30 years of experience in the development of automation algorithms for various systems for scientific research. She is a coauthor of more than 30 peer-review papers and patents.

Egor Sergeev (student) is a laborant at the Laboratory of Biophotonics of Institute of Applied Physics of Russian Academy of Sciences.

Valeria Perekatova received her master's degree in physics in 2015 from Lobachevsky State University of Nizhni Novgorod. She is a junior researcher at the Laboratory of Biophotonics of Institute of Applied Physics of Russian Academy of Sciences. Her current research focuses on the developing the methods of optoacoustic imaging of biological tissues. She is the coauthor of eight peer-review articles and conference proceedings related to optoacostics.

Anna Orlova received her PhD in biology from Nizhny Novgorod State University in 2004. She is a senior scientist at the Laboratory of Biophotonics of the Institute of Applied Physics RAS. Major area of her research focuses on optical imaging both in clinical and experimental oncology. She is the coauthor of more than 30 peer-review articles and several book chapters.

Valentina Kotomina is the head at the Laboratory for surface preparation of semiconductors and solids at the Research Institute for Physics and Technology. Major area of her research focuses on highmolecular compounds and photolitographic etching. She is a coauthor of more than 15 peer-review articles and several book chapters.

Ilya Turchin $(\mathrm{PhD})$ is the head of the Department for radiophysics methods in medicine at the Institute of Applied Physics of RAS and is an expert in optical imaging systems design (optical diffuse imaging, fluorescence imaging, photoacoustics) and biomedical applications. He has over 10 years of experience as a primary investigator in optical imaging, photodynamic therapy, and translation of optical techniques into clinics. He has published more than 50 peer-review articles. 